\title{
A switchable digital microfluidic droplet dye-laser $\dagger$
}

\author{
Alexander J. C. Kuehne, ${ }^{a}$ Malte C. Gather, ${ }^{b}$ Irwin A. Eydelnant, ${ }^{c}$ Seok-Hyun Yun, ${ }^{b}$ David A. Weitz ${ }^{a d}$ \\ and Aaron R. Wheeler *ce
}

\author{
Received 11th May 2011, Accepted 29th July 2011 \\ DOI: 10.1039/c1lc20405j
}

\begin{abstract}
Digital microfluidic devices allow the manipulation of droplets between two parallel electrodes. These electrodes can act as mirrors generating a micro-cavity, which can be exploited for a droplet dye-laser. Three representative laser-dyes with emission wavelengths spanning the whole visible spectrum are chosen to show the applicability of this concept. Sub-microlitre droplets of laser-dye solution are moved in and out of a lasing site on-chip to down-convert the UV-excitation light into blue, green and red laser-pulses.
\end{abstract}

\begin{abstract}
Miniaturization of optical components is a key requirement for interfacing light sources, sensors and other optical equipment with lab-on-chip devices. ${ }^{1}$ For example, lasers have been combined with microfluidic devices, ${ }^{2}$ however conventional lasers are only capable of producing a few discrete wavelengths. To probe different fluorescent markers or samples for example in cell sorting, immunoassays and DNA-sequencing, it is desirable to access multiple narrow and coherent excitation wavelengths. ${ }^{3}$ Optical parametric oscillators (OPOs) or optical cavities filled with dye solutions are typical means to vary the wavelength of a laser and afford a wide spectrum of excitation wavelengths; however, the cost and size of OPOs restricts them from lab-onchip integration. There are a few reports of miniaturized dye lasers in the literature, ${ }^{4}$ none of which allows instantaneous control over the emission wavelength together with a directed emission beam.

In this paper, we present a new approach to the miniaturization of dye-lasers, in which fast switching of the emission wavelength is achieved by using digital microfluidics (DMF). The emission wavelength of the DMF dye laser can be changed by moving droplets of different dye solutions in and out of an excitation beam, while the geometry of the DMF device allows directed emission of the resulting laser beam.
\end{abstract}

${ }^{a}$ School of Engineering and Applied Sciences, Harvard University, 9 Oxford St, Cambridge, MA, 02138, USA

${ }^{b}$ Harvard Medical School and Wellman Center for Photomedicine, Massachusetts General Hospital, 50 Blossom St, Boston, MA, 02114, USA 'Institute for Biomaterials and Biomedical Engineering, University of Toronto, 164 College St, Toronto, ON, M5S 3G9, Canada. E-mail: aaron.wheeler@utoronto.ca; Fax: +416946 3865; Tel: +416 9463864

${ }^{d}$ Department of Physics, Harvard University, 9 Oxford St, Cambridge, MA, 02138, USA

${ }^{e}$ Department of Chemistry, University of Toronto, $80 \mathrm{St}$. George St, Toronto, ON, M5S 3H6, Canada

$\dagger$ Electronic Supplementary Information (ESI) available: Experimental details. See DOI: $10.1039 / \mathrm{c} 1 \mathrm{lc} 20405 j$
In the DMF two-plate geometry, droplets are sandwiched between a bottom substrate bearing an array of patterned electrodes coated by an insulator, and a top substrate bearing a counter-electrode, as depicted in Fig. 1. Droplets in such devices move by electrodynamic forces: when a voltage is applied to an electrode adjacent to a droplet, electric forces act on free charges in the droplet meniscus of conductive liquids or dipoles inside dielectric liquids. With sequential actuation of electrodes, droplets can be dispensed and moved from electrode to electrode across the entire array on the bottom-plate. ${ }^{5,6}$ Digital microfluidics and related techniques have been used for a number of different optical applications, ${ }^{7}$ but DMF has so far never been used to form lasers or optical cavities. Digital microfluidic devices can be readily adapted to form a microfluidic dye laser when the parallel electrode-plates are used as mirrors to form an optical resonator. Droplets containing laser-dyes act as the gainmedium. Upon optical excitation by a focussed pump laser, the dye-loaded droplets generate fluorescence which is reflected back into the gain medium by the adjacent electrodes. This provides

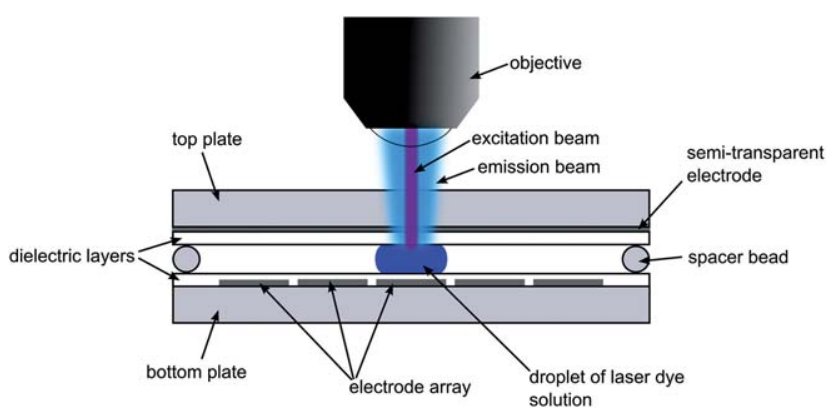

Fig. 1 Schematic of a DMF dye-laser with a reflective array of electrodes on the bottom-plate and a semi-reflective counter-electrode on the top plate. The two plates form the optical microcavity. One droplet is shown, containing a blue laser-dye, which is being excited by a UV laser beam (purple) at the dedicated lasing site. 
the necessary feedback and leads to amplification of the fluorescence by stimulated emission. Since the top-electrode is partially transparent in these devices, light can leak from the optical micro-cavity, resulting in directed laser-emission. The wavelength of the generated laser light is determined by the fluorescence spectrum of the laser dye. The DMF device thus allows facile tuning of the laser wavelength by moving droplets loaded with different dyes into the focus of the pump laser.

To ensure good optical characteristics of the laser cavity, the electrodes are manufactured from silver, which provides high reflectivity and low optical losses compared to other metals. The silver electrode on the top-plate has a thickness of only $30 \mathrm{~nm}$ resulting in a semi-transparent mirror to allow $5-20 \%$ transmission of the excitation laser beam and the emitted laser light (see Fig. S1 in the electronic supplementary information $\dagger$ ). While DMF electrodes are often coated with Parylene-C as a dielectric barrier followed by Teflon ${ }^{\circledR}$ AF spin-coating for hydrophobicity ${ }^{6}$ in this work, these two layers are replaced with a single layer of Teflon ${ }^{\circledR}$ FEP film. This is important, as Parylene-C is prone to photo-oxidation on prolonged UV exposure. ${ }^{8}$ This strategy is also advantageous because films can be removed and replaced, prolonging device lifetimes. ${ }^{9}$ Commercially available $100 \mu \mathrm{m}$ diameter silica spheres are applied on the edges of the device as precise spacers to hold the two plates apart, resulting in a total cavity length of $\sim 126 \mu \mathrm{m}$ when accounting for the $12.7 \mu \mathrm{m}$ thick fluoropolymer films.

Four rows of electrodes with adjacent fluid reservoir-electrodes form the bottom-plate layout. Each electrode can be individually actuated via an electrode pad, as indicated in Fig. 2. The unpatterned top-plate is aligned so that it covers the set of electrodes and is flush with the edges of the reservoir-electrodes. This way the reservoirs can be filled with the laser-dye solutions by exploiting capillary forces. The laser-dyes coumarin-450 (C450), pyrromethene-556 (PM-556) and 4-(dicyanomethylene)-2methyl-6-(4-dimethylaminostyryl)-4H-pyran (DCM) are used. C-450 and DCM are dissolved in DMSO, whereas PM-556 is dissolved in water, illustrating the flexibility of the DMF approach for different solvents. Laser-dye droplets are dispensed from the reservoir-electrodes onto an array of actuation electrodes, and driven to the designated laser site (an electrode in the center of the array), as illustrated in Fig. 2.

The $355 \mathrm{~nm}$ line of a frequency tripled, Q-switched Nd:YAG laser serves as the optical pump beam, and is focussed into the space above the lasing site through the semi-transparent topplate. Droplets loaded with different laser-dye solutions are moved in and out of the lasing site, and the resulting emission beam is collected by the same optics, separated from the pump beam and guided to a spectrograph. The lasing and fluorescence spectra of all of the dye-droplets show a fine modulation, as can be seen in Fig. 3. This series of peaks corresponds to the subsequent longitudinal modes of the DMF-micro-cavity. The cavity length of the DMF-device can be extracted from this modespacing by using Eqn (1)

$$
L \approx \frac{\lambda^{2}}{2 \Delta \lambda n} \text { for } \lambda \gg \Delta \lambda
$$

where $L$ is the micro-cavity length, $\lambda$ is the emission wavelength, $\Delta \lambda$ is the wavelength separation between the peaks and $n$ the average refractive index of the gain medium. Using literature

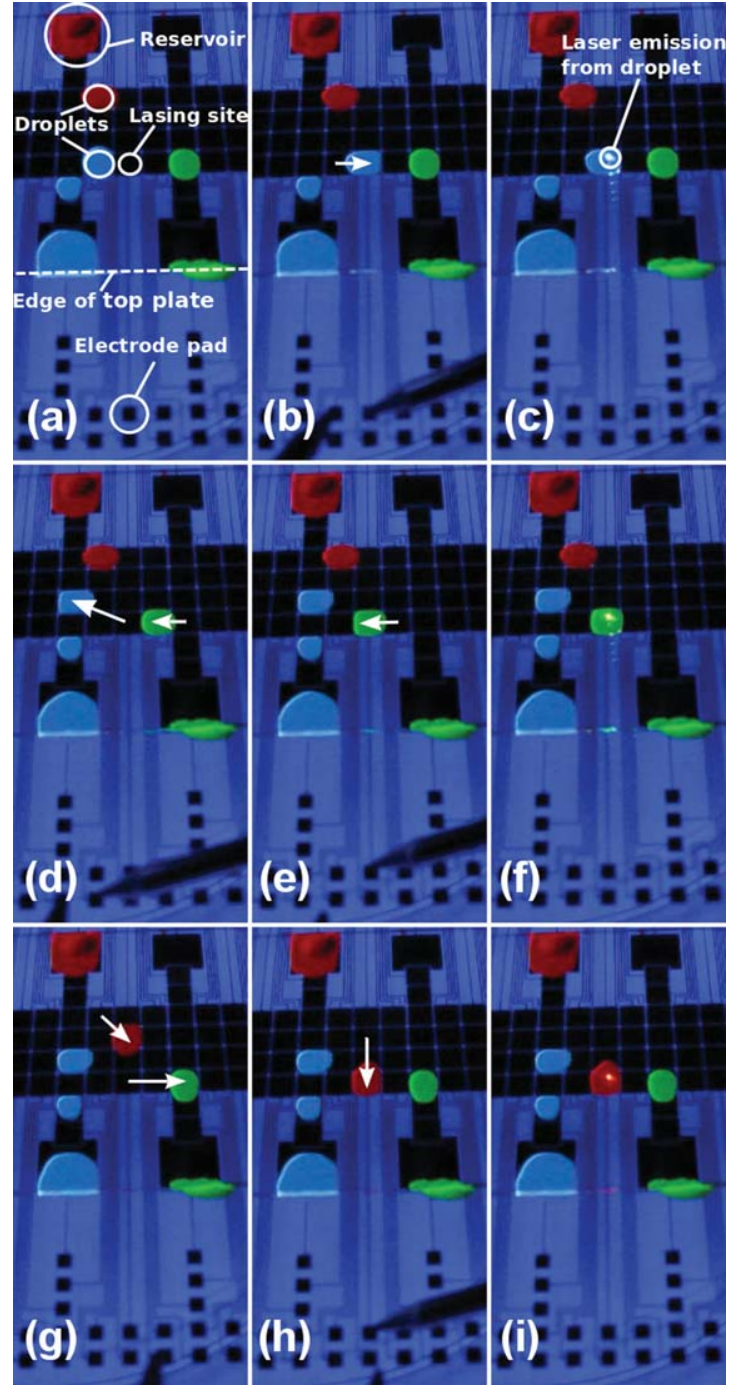

Fig. 2 Frames from a movie depicting device operation (available in the online supplementary information $\dagger$ ), collected using low intensity blacklight to make the droplets visible. Each droplet has a cross-sectional area of approximately $2.2 \times 2.2 \mathrm{~mm}$. In (a-c), a droplet of the blue emitting laser dye C-450 is moved onto the dedicated lasing electrode. In (d-f) the blue emitting droplet is moved away and a droplet of green emitting laser dye PM-556 is moved onto the lasing electrode. In ( $\mathrm{g}-\mathrm{i})$ the green emitting droplet is moved back to the reservoir and the droplet of red emitting DCM laser dye is moved onto the dedicated lasing site.

values of $n_{\text {water }}=1.333$ and $n_{\text {DMSO }}=1.479$, cavity lengths of $120 \mu \mathrm{m}$ to $125 \mu \mathrm{m}$ are obtained, which suggests that the silica beads used to separate the two plates of the device may partially indent the soft fluoropolymer coating on the electrodes.

To determine the lasing-threshold for each of the three dye droplet systems, the pump energy is gradually increased and the output spectrum is recorded. To obtain the output power at different pump energies, the area under each output spectrum is integrated. At low pump energies the output power increases slowly with increasing pump energy. At a certain pump energy, however, the slope of the output increases steeply, which marks the lasing threshold, as shown in Fig. 4. When pumped above threshold, the initially broad emission spectrum narrows into distinct laser modes, as can be seen in Fig. 3b. The threshold 

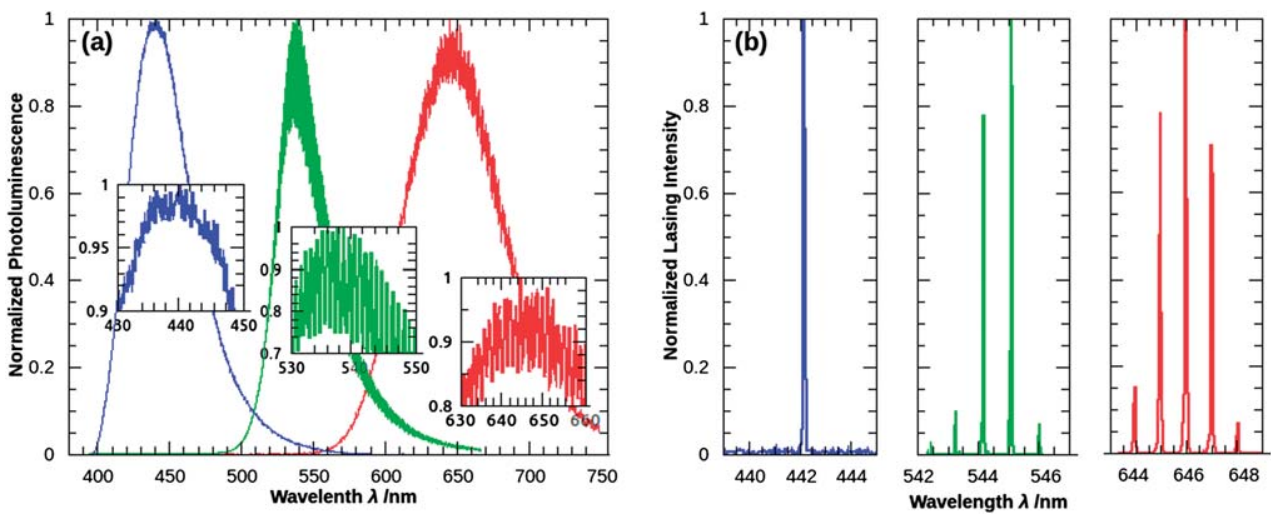

Fig. 3 (a) Normalized photoluminescence generated from dye-droplets located inside the DMF cavity. Insets show close-ups of the modulation at the maxima of the photoluminescence spectra. (b) Lasing spectra of the three dye-droplets. C-450 (blue), PM-556 (green) and DCM (red).

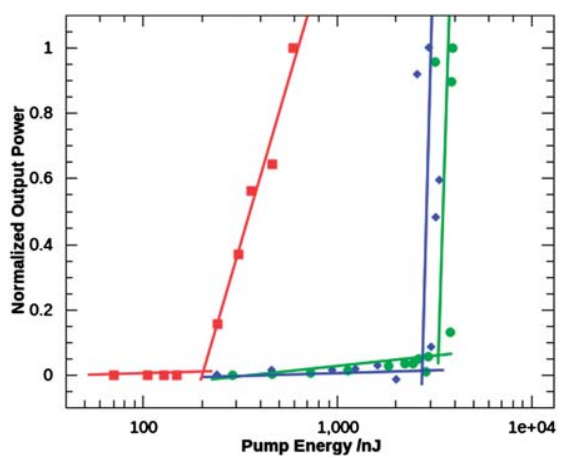

Fig. 4 Plot of the normalized output power as a function of the laser pump energy for droplets of C-450 (blue diamonds), PM-556 (green circles) and, DCM (red squares). The solid symbols are experimental data, and the lines were added as a guide to the eye.

energy for DCM is significantly lower than those for C-450 and PM-556. This can be explained by the wavelength-dependent reflectivity of the silver electrodes, which provide higher reflectivity $(>95 \%)$ at wavelengths above $600 \mathrm{~nm}$ than in the blue and green parts of the spectrum (88-93\%); see Fig. S1 in the online supplementary information. $\dagger$ Hence the quality of the resonator is better for the emission wavelengths of DCM than for those of C-450 and PM-556, leading to a lower threshold for the red emitting dye.

For a droplet of C-450 in the DMF micro-cavity a single laserline is generated for excitation above threshold, while for PM556 and DCM a set of four or five laser lines are observed respectively, as shown in Fig. 3b. The laser lines of all three dyes have a full width at half maximum (FWHM) of less than $0.1 \mathrm{~nm}$. The laser-dye emission is directed perpendicular to the electrodes and emitted through the semi-transparent electrode, as opposed to previous demonstrations of droplet-based lasers, which operated via whispering gallery mode resonance and hence provided no directed emission. ${ }^{2}$ For whispering gallery mode laser emission is not directed towards a specific probe location, which limits the applicability of such laser geometries.

The DMF platform can be interfaced with other devices by using the top-plate as the substrate. ${ }^{10}$ DMF droplet dye lasers therefore show potential as integrated switchable laser sources for other microfluidic or microelectromechanical systems. We propose that in the future, a larger chip design might be used to control a large number of different laser dyes which could cover virtually any desirable wavelength range. Furthermore, both electrodes can be made semi-transparent to increase functionality of the device. The metal electrodes could be replaced by indium tin oxide (ITO) coated with dielectric mirrors, which would lead to less losses and higher efficiencies. To replace the bulky excitation source, on-chip micro UV-LEDs could be used to pump the droplets. ${ }^{11}$ Thus, we posit that the new DMF droplet dye laser format is extremely versatile and may be useful for a wide range of applications requiring on-chip optical sensing.

\section{Acknowledgements}

This work was supported by the NSF (DMR-1006546) and the Harvard MRSEC (DMR-0820484). This work was performed in part at the Harvard Center for Nanoscale Systems (CNS), a member of the National Nanotechnology Infrastructure Network (NNIN), which is supported by the NSF (ECS0335765) and in part at the Wellman Center with support by the NSF (ECCS-0801412). A.J.C.K. thanks the DAAD for financial support. M.C.G. acknowledges financial support from the Bullock-Wellman Fellowship. I.A.E. thanks the natural sciences and engineering research council of Canada for a graduate fellowship. A.R.W. thanks the Canada Research Chair program for a CRC.

\section{References}

1 B. Ibarlucea, E. Fernandez-Rosas, J. Vila-Planas, S. Demming, C. Nogues, J. A. Plaza, S. Bttgenbach and A. Llobera, Anal. Chem., 2010, 82, 4246.

2 D. Psaltis, S. R. Quake and C. Yang, Nature, 2006, 442, 381; C. Monat, P. Domachuk and B. J. Eggleton, Nat. Photonics, 2007, $1,106$.

3 E. Brouzes, M. Medkova, N. Savenelli, D. Marran, M. Twardowski, J. B. Hutchison, J. M. Rothberg, D. R. Link, N. Perrimon and M. L. Samuels, Proc. Natl. Acad. Sci. U. S. A., 2009, 106, 14195; K. B. Mogensen and J. P. Kutter, Electrophoresis, 2009, 30, S92; M. C. Gather and S. H. Yun, Nat. Photonics, 2011, 5, 406; Y. Chen, L. Lei, K. Zhang, J. Shi, L. Wang, H. Li, X. M. Zhang, Y. Wang and H. L. W. Chan, Biomicrofluidics, 2010, 4, 043002.

4 S. K. Y. Tang, Z. Li, A. R. Abate, J. J. Agresti, D. A. Weitz, D. Psaltis and G. M. Whitesides, Lab Chip, 2009, 9, 2767; H. Li, L. Lei, Q. Zeng, J. Shi, C. X. Luo, H. Ji, Q. Ouyang and Y. Chen, Sens. Actuators, B, 2010, 145, 570; S. K. Y. Tang, R. Derda, Q. Quan, M. Lončar and 
G. M. Whitesides, Opt. Express, 2011, 19, 2204; B. Helbo, A. Kristensen and A. Menon, J. Micromech. Microeng., 2003, 13, 307; G. Aubry, Q. Kou, J. Soto-Velasco, C. Wang, S. Meance, J. J. He and A. M. Haghiri-Gosnet, Appl. Phys. Lett., 2011, 98, 111111. 5 M. Abdelgawad and A. R. Wheeler, Adv. Mater., 2009, 21, 920.

6 D. Chatterjee, B. Hetayothin, A. R. Wheeler, D. J. King and R. L. Garrell, Lab Chip, 2006, 6, 199.

7 S. Yang, K. Zhou, E. Kreit and J. Heikenfeld, Appl. Phys. Lett., 2010, 97, 143501; R. A. Hayes and B. J. Feenstra, Nature, 2003, 425, 383; B. Berge and J. Peseux, Euro. Phys. J. E, 1999, 3, 159.
8 J. B. Fortin and T.-M. Lu, Thin Solid Films, 2001, 397, 223; K. G. Pruden, K. Sinclair and S. Beaudoin, J. Polym. Sci., Part A: Polym. Chem., 2003, 41, 1486.

9 H. Yang, V. N. Luk, M. Abelgawad, I. Barbulovic-Nad and A. R. Wheeler, Anal. Chem., 2009, 81, 1061.

10 M. W. L. Watson, M. J. Jebrail and A. R. Wheeler, Anal. Chem., 2010, 82, 6680 .

11 H. X. Zhang, D. Massoubre, J. McKendry, Z. Gong, B. Guilhabert, C. Griffin, E. Gu, P. E. Jessop, J. M. Girkin and M. D. Dawson, Opt. Express, 2008, 16, 9918. 